\title{
Associations between Sensorimotor, Autonomic and Central Neuropathies in Diabetes Mellitus
}

\author{
Christina Brock $^{1^{*}}$, Eirik Søfteland ${ }^{2,3}$, Jens Brøndum Frøkjær ${ }^{4,5}$, Asbjørn Mohr Drewes ${ }^{1,5}$ and Lars Arendt-Nielsen ${ }^{6}$ \\ ${ }^{1}$ Mech-Sense, Department of Gastroenterology, Aalborg University Hospital, Denmark \\ ${ }^{2}$ Department of Medicine, Haukeland University Hospital, Bergen, Norway \\ 3 Institute of Medicine, University of Bergen, Norway \\ ${ }^{4}$ Mech-Sense, Department of Radiology, Aalborg University Hospital, Aalborg, Denmark \\ ${ }^{5}$ Departments of Clinical Medicine, Aalborg University, Aalborg, Denmark \\ ${ }^{6}$ Center for Sensory-Motor Interaction (SMI), Department of Health Science and Technology, Faculty of Medicine, Aalborg University, Denmark
}

"Corresponding author: Christina Brock, DVM, Ph.D, Mech-Sense, Department of Gastroenterology Aalborg University Hospital Mølleparkvej 4 DK-9000 Aalborg, Denmark, Tel: +45 9766 3523; Fax: +45 9932 6507; E-mail: cb@mech-sense.com

Rec date: Apr 09, 2014, Acc date: Jun 06, 2014, Pub date: Jun 10, 2014

Copyright: (c) 2014 Brock C, et al. This is an open-access article distributed under the terms of the Creative Commons Attribution License, which permits unrestricted use, distribution, and reproduction in any medium, provided the original author and source are credited.

\begin{abstract}
Background: Patients with long-standing diabetes mellitus (DM) are exposed to hyperglycemia and metabolic disorders associated with extensive neuronal damage. Using a bedside applicable setup, the aim was to explore whether diabetes patients suffering from sensorimotor neuropathy had co-existing autonomic and central neuropathy.
\end{abstract}

Methods: Twenty DM patients (10 women, average age $58.3 \pm 12.0$ years) with sensorimotor polyneuropathy and 16 healthy volunteers (HV) $(9$ women, age $62.6 \pm 10.5$ years) were recruited. Heart rate variability was recorded. Peripheral tactile detection threshold and sensation to pinprick was assessed by Von Frey-filaments. Sensitivity to pressure of the forearm extensor digitorum muscle was measured before and after conditioning pain modulation (CPM) induction by immersing the contra lateral hand into ice water in 180 seconds.

Results: In comparison to HV, DM patients had lower variance $(7.1 \pm 5.6$ vs. $13.3 \pm 8.1$ beats pr. min.; $P=0.02)$ and mean standard deviation $(3.3 \pm 2.0$ vs. $5.6 \pm 2.8 ; \mathrm{P}=0.01)$ of heart rate. They also had peripheral hypoesthesia to tactile stimulation: Median ( $3.0 \mathrm{~g}(0.8-12.5)$ vs. $1.0 \mathrm{~g}(0.4-1.4), \mathrm{P}=0.03)$ and less efficacious CPM (13.9 $\pm 14.8 \%$ vs. $37.4 \% \pm 28.9 \% ; P=0.005)$. In patients, peripheral hypoesthesia was associated to mean heart rate $(P=0.01)$, standard deviation of heart rate $(P=0.004)$, and to CPM $(P=0.046)$.

Conclusion: DM patients with sensorimotor neuropathy showed generalized polyneuropathy evident as peripheral hypoesthesia, autonomic neuropathy and impaired CPM. Von Frey tactile detection threshold was associated with heart rate variability and CPM. The clinical approach and combined testing procedures, may serve as a prognostic platform to quantitatively evaluate the severity, extension and progression of diabetic neuropathy.

Keywords: Diabetes mellitus; Hypoesthesia; Autonomic neuropathy; Conditioning pain modulation

\section{Introduction}

Diabetes Mellitus (DM) is a group of metabolic diseases characterized by hyperglycemia resulting from defects in insulin secretion, insulin action, or both. Chronic hyperglycemia is associated with long-term damage, dysfunction and failure of various organs, especially targeting the eyes, kidneys, heart, blood vessels and the nerves [1]. Hence, severe peripheral sensorimotor, autonomic and central neuropathies are common complications to longstanding DM. Advanced peripheral diabetic neuropathy classically manifests as chronic progressive thick $(A \beta)$ and thin $(A \delta)$ fiber neuropathy affecting myelinated axons and later affecting also the unmyelinated $\mathrm{C}$-fibers [2,3]. The natural history of the disease varies from intermittent mild symptoms to manifest neuropathic pain, the latter being present in up to one fourth of the patients diminishing their quality of life [4-6]. The typical appearance is length dependent sensorimotor neuropathy caused by chronic progressive symmetric deafferentation and Schwann cell degeneration affecting axons of the distal lower extremities [2,7]. Sensorimotor neuropathy can be reliably assessed by use of vibration assessment, quantitative sensory testing measuring decreased sensitivity to pinprick and von Frey tactile detection threshold and electrophysiological techniques determining alterations of sensory nerve conduction velocity [8].

Accurate detection and quantification of small fiber neuropathy in the skin is challenging, even though different methods are available. Quantitative sensory testing are limited by involvement of larger fibres, however the more objective assessments including skin biopsies quantifying nerve fiber density or corneal confocal microscopy, are limited to specialized centers [9-11]. Diabetic autonomic neuropathy shares same etiology as peripheral neuropathy. Clinically autonomic neuropathy can be present with or without presence of large fiber neuropathy. Symptoms are often mild; nevertheless it has been shown that approximately $50 \%$ of diabetes type 1 patients and $75 \%$ of diabetes 
Page 2 of 6

type 2 patients had subtle objective autonomic impairments [12]. Generalized autonomic disease, resulting in a variety of troublesome symptoms including orthostatic hypotension, nausea, abnormal gastrointestinal motility and erectile dysfunction was reported in $10 \%$. A common approach to assess the generalized severity of autonomic neuropathy is based on cardiac Heart Rate Variability (HRV), which is diminished in autonomic neuropathy.

The pain systems exert specialized descending Conditioning Pain Modulation (CPM) from the brainstem and supra-spinal structures, which modulates the upstream afferent transmission. The CPM efficacy is likely related to the generalized status of the central nervous system, and hence diabetes induced central impairment may likely interfere with this complex network [13-15]. Changes in pain modulation processes, as reflected by dynamic psychophysical tests, are now increasingly recognized as clinically relevant. Hence, CPM has been shown to predict the efficacy of duloxetine in treatment of diabetic neuropathy [16]. However, a comprehensive neurophysiological profile including the different levels and the interaction between them has not been studied in DM patients with peripheral neuropathy. Thus, in order to determine the degree of generalized neuropathy in DM patients, we established a clinical applicable platform providing the possibility of investigating coexisting sensorimotor, autonomic and central neuropathies.

We hypothesized that DM patients, due to generalized neuropathy, would present with coexisting sensorimotor, autonomic and central neuropathy. Hence, the specific aims were to compare DM patients with Healthy Volunteers (HV) in terms of 1) tactile detection and pain to pinprick using von Frey monofilaments, 2) assessment of heart rate variability, 3) CPM, and 4) the interaction between the different parameters.

\begin{tabular}{|c|c|c|c|c|}
\hline & \multirow{2}{*}{ Variables } & Patients & Healthy volunteers & \multirow{2}{*}{ Significance } \\
\hline & & $(n=20)$ & $(n=16)$ & \\
\hline \multirow{7}{*}{ Basic data } & Age (years) & $58.3( \pm 12.0)$ & $62.6( \pm 10.5)$ & NS \\
\hline & Sex (male/female) & $10 / 10$ & $7 / 9$ & NS \\
\hline & Body Mass Index kg/m2 & $28.0( \pm 3.62)$ & $25.8( \pm 3.38)$ & NS \\
\hline & Diabetes duration (years) & $15.8( \pm 10.0)$ & - & - \\
\hline & $\mathrm{HbA} 1 \mathrm{c}(\%)$ & $8.0( \pm 1.0)$ & - & - \\
\hline & Hypertension\% & 81 & 19 & $P=0.001$ \\
\hline & Beta-blocker (\%) & 27 & 0 & $P=0.04$ \\
\hline \multirow[t]{4}{*}{ Sensorimotor neuropathy } & First sensation Von Frey (g) & *3.0 (0.8-12.5) & *1.0 (0.4-1.4) & $P=0.03$ \\
\hline & Sensory score (VAS) to 26 grinprick & & $2.6 \pm 1.3$ & $P=0.10$ \\
\hline & \multirow{2}{*}{ Muscle pressure (KPa) } & $1.9( \pm 0.8)$ & \multirow{2}{*}{$283 \pm 110$} & \multirow{2}{*}{$P=0.12$} \\
\hline & & $358 \pm 171$ & & \\
\hline \multirow[t]{5}{*}{ Autonomous neuropathy } & Mean heart rate variability & $7.1 \pm 5.6$ & $13.3 \pm 8.1$ & $P=0.02$ \\
\hline & Mean SD of heart rate & $3.3 \pm 2.0$ & $5.6 \pm 2.8$ & $P=0.01$ \\
\hline & LF norm & *44.3 (21-76) & $71.2(57-87)$ & $P=0.04$ \\
\hline & HF norm & *55.7 (24-79) & $28.8(13-43)$ & $P=0.04$ \\
\hline & $\mathrm{LF} / \mathrm{HF}$ & ${ }^{*} 0.8(0.27-3.3)$ & $2.47(1.3-6.8)$ & $P=0.04$ \\
\hline \multirow[t]{2}{*}{$\begin{array}{l}\text { Conditioning } \\
\text { modulation }\end{array}$} & Absolute increase after CPM $(\mathrm{kPa}$ & $44 \pm 69$ & $102 \pm 73$ & $P=0.02$ \\
\hline & Relative increase after CPM (\%) & $14 \pm 15$ & $37 \pm 29$ & $\mathrm{P}=0.005$ \\
\hline
\end{tabular}

Table 1: Subject characteristics, Patient characteristics, presented as means ( \pm standard deviation), except Von Frey marked with a ${ }^{*}$ indicating data presented as median (inter quartile range).

\section{Methods}

\section{Subjects}

Data were collected from June 2010 until October 2011, and data regarding visceral, cardiac and sensorimotor neuropathies have been reported previously by Søfteland et al. [17]. Patient characteristics are presented in Table 1 . Twenty DM patients (10 women, average age $58.3 \pm 12.0$ years) with clinical suspicion of sensorimotor neuropathy (i.e. symptoms and abnormal pinprick test) defined according to Boulton et al. [18], were recruited from Haukeland University Hospital, Bergen, Norway and St. George Hospital, Székesfehérvár, Hungary. Mean glycated hemoglobin (HbAlc) was $8.0 \pm 1.0 \%(64$ 
$\mathrm{mmol} / \mathrm{mol}$ ). Regular pain-modifying medications were paused minimum 24 hours prior to experimental testing.

For comparison sixteen HV, matched for age and gender ( 9 women, age $62.6 \pm 10.5$ years) were recruited in Bergen through newspaper advertisement. Subjects were examined by a doctor to rule out any disease. Oral and written informed consent was obtained from all participants, and the study was approved by the local ethical committees at Haukeland and St. George Hospitals. Experiments were carried out according to the Helsinki declaration.

\section{Experimental protocol}

All patients and HV fasted for 6 hours. To standardize influence of glucose and insulin levels on sensory assessments, a hyperinsulinemiceuglycemic clamp technique ensured continuous adjustment of the blood glucose level to 5-6 $\mathrm{mmol} / \mathrm{L}$ throughout the entire study procedure.

Most sensory afferents likely encode both non-painful and painful sensations. Hence, we instructed subjects in use of the modified 0-10 electronic Visual Analogue Scale (VAS), which has been widely used in sensory experiments [19]. The use of VAS was facilitated through anchor words, where $0=$ no perception; $1=$ first perception, $3=$ vague perception of moderate sensation; $5=$ pain detection threshold; $7=$ moderate pain and $10=$ worst perceivable pain. VAS was recorded continuously during testing.

\section{Von frey tactile detection threshold}

Von Frey filaments (Marstock Nervtest, Schriesheim, Germany) made of optic glass fibers, are highly elastic and bends to a certain pressure. Endings are epoxy coated to ensure constant contact surface for fibers with different diameters. Subjects were tested at the base of the dorsum of the first toe on the dominant side and the weight (i.e. thickness of the filament) corresponding to tactile detection threshold was noted.

\section{Sensory response to pinprick}

Von Frey filaments with the size of 26 gram were also used to assess the sensory score to a pinprick.

\section{Autonomic nervous system tests}

The autonomic nervous system was investigated by examining heart rate variability using the Heart Rhythm Scanner PE (Biocom Technologies, Poulsbo, WA, USA). The system investigates both timeand frequency domain measures of the HRV and its use has been described and validated elsewhere [20]. Data are presented in Table 1. Mean variance of heart rate at rest (beats pr. minute (bpm) pr. ms) and standard deviation of heart rate (consecutive RR intervals) was used for further analysis.

\section{Efficacy of descending pain modulation}

Noxious heterotopic conditioning painful stimulus was applied by immersing the right hand until the wrist in cooled circulated water (2 $\pm 0.3^{\circ} \mathrm{C}$ ), the so-called cold pressor test. Study subjects were encouraged to withstand the 180 seconds immersion, however instructed to remove the hand, if pain was considered intolerable. A test stimulus was applied before and immediately after the cold pressor test consisting of: Pressure tolerance threshold ( 7 on the visual analogue scale) assessed in the mid part of the extensor digitorum muscle $10 \mathrm{~cm}$ distal from the left elbow joint, by use of a handheld electronic pressure algometer with a standard probe of $1 \mathrm{~cm} 2$ (Somedic AB, Stockholm, Sweden). The pressure was increased with a rate of $30 \mathrm{kPa} / \mathrm{s}$. The efficacy of the CPM was presented as absolute $(\mathrm{kPa})$ and relative (\%) change in muscle sensitivity.

\section{Statistical Analysis}

Descriptive data are presented as mean with Standard Deviation (SD) or median with Inter-Quartile Range (IQR) where appropriate. To test differences between patients and HV, comparison of demographics, HRV and glucose levels were done by Student's t-tests. Von Frey filament size between patients and HV were compared with a Kruskal-Wallis One-Way Analysis Of Variance (ANOVA) as data were not normally distributed. To compare efficacy of descending pain modulation between patients and HV a one way ANOVA was used. Correlation analyses were done using Spearman's test to investigate associations between peripheral hypoesthesia, HRV and CPM efficacy. The software package Sigma Stat v.3.0 (SPSS Inc., Chicago; IL, USA) was used in the analysis and $\mathrm{P}$-values $\leq 0.05$ were considered significant.

\section{Results}

All subjects underwent the hyperinsulinemic clamp without any adverse events. No difference between patients and HV in clamped mean glucose levels: $6.1 \pm 1.1$ vs. $5.5 \pm 0.6 \mathrm{mmol} / \mathrm{L}(\mathrm{P}=0.2)$ was found

\section{Von Frey tactile detection threshold}

Patients reported higher weight of von Frey filament upon first sensation compared to HV: Median $3.0 \mathrm{~g}$ (Inter Quartile Range (IQR): $0.8-12.5)$ vs. $1.0 \mathrm{~g}$ (IQR: $0.4-1.4),(\mathrm{H}=4.9 ; \mathrm{P}=0.03)$, indicating thick fiber impairment in DM patients (Figure 1).

\section{Sensory response to pinprick with 26 gram Von Frey filament}

There was no difference between patients and HV in the sensory response to pinprick with a 26 gr monofilament: Mean VAS of $1.9 \pm$ 0.8 vs. $2.6 \pm 1.3(\mathrm{P}=0.1)$.

\section{Assessments of autonomic nervous system}

Patients had lower mean variance of heart rate $(7.1 \pm 5.6$ vs. $13.3 \pm$ 8.1 beats pr. minute/ms, $\mathrm{P}=0.02$ ) and mean standard deviation of heart rate $(3.3 \pm 2.0$ vs. $5.6 \pm 2.8 \mathrm{P}=0.01)$ in comparison to $\mathrm{HV}$, indicating autonomic neuropathy in $\mathrm{DM}$ patients.

\section{Cold pressor test}

All subjects tolerated immersion of the hand into cooled water for 180 seconds. However, one patient was withdrawn due to a severe vaso-vagal response immediately after the cold pressor test. Thus, data analyses were obtained in 19 patients. No difference between patients and $\mathrm{HV}$ was seen in mean pain perception of the cold pressor test: VAS score of $5.3 \pm 2.0$ vs. $6.1 \pm 2.0(\mathrm{P}=0.2)$. 


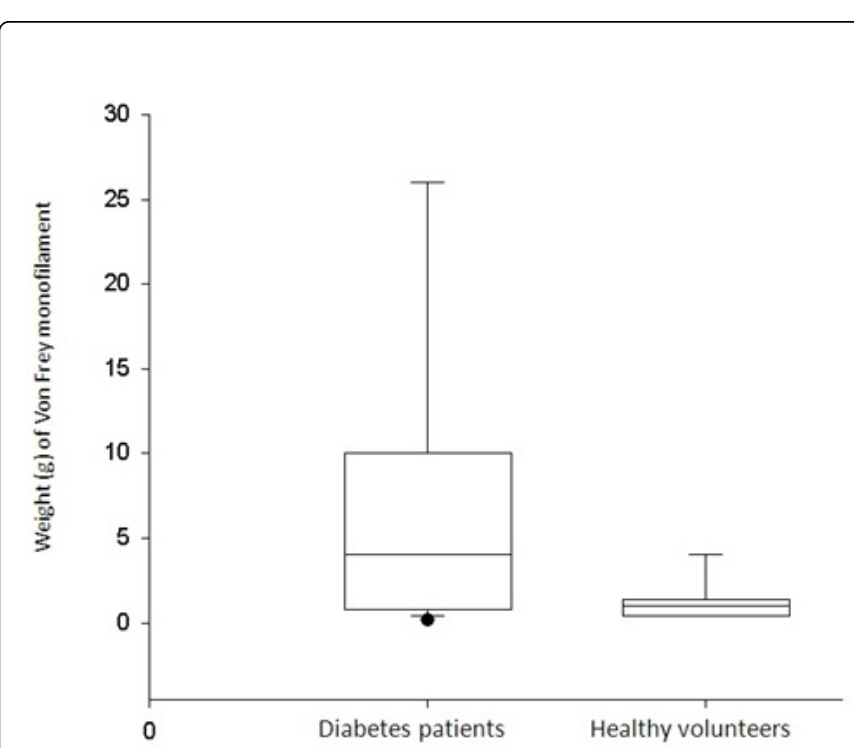

Figure 1: Box-plots showing the differences weight of the von Frey monofilament needed to evoke in first sensations in patients and healthy volunteers. The variability in the dataset is larger in the patient group, indicating different degrees of peripheral hypoesthesia.

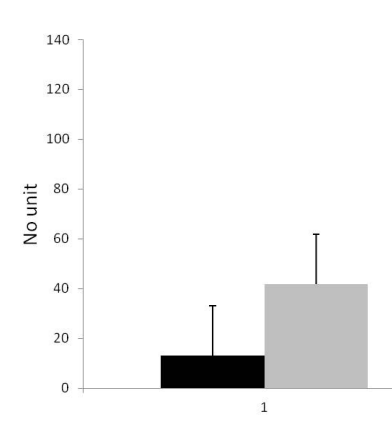

Diabetes patient

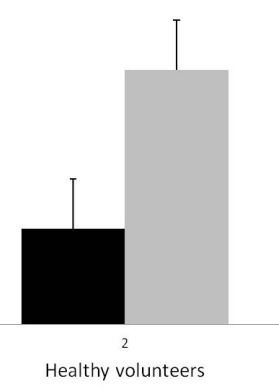

Figure 2: In comparison to healthy volunteers, diabetes patients showed decreased relative (\%) efficacy of the descending pain modulation (black) and a non-dynamic appearance of the difference in absolute values $(\mathrm{kPa})$ of the tolerated muscle pressure in the extensor digitorum muscle (grey) before and after immersing the contra-lateral hand into ice water, indicating impairment and dysfunctional central pain modulation.

\section{Efficacy of descending pain modulation}

No difference between patients and $\mathrm{HV}$ was seen in tolerated muscle pressure before the cold pressor test: $357.6 \pm 171.0$ vs. $282.9 \pm$ $109.6 \mathrm{kPa},(\mathrm{F}=3.0 ; \mathrm{P}=0.12)$ (Figure 2).

Pressure tolerance threshold increased in both groups following $\mathrm{CPM}$, however significantly less (non-dynamic) in patients than in $\mathrm{HV}: 43.9 \pm 68.8$ vs. $102.1 \pm 73.3 \mathrm{kPa}(\mathrm{F}=5.9 ; \mathrm{P}=0.02)$ (Figure 2). Correspondingly, relative pressure sensitivity increased in both groups, however less efficacious sin patients: $13.9 \pm 14.8 \%(\mathrm{DM})$ vs.
$37.4 \% \pm 28.9 \%(\mathrm{HV})(\mathrm{F}=9.2 ; \mathrm{P}=0.005)$. Both indicate impaired $\mathrm{CPM}$ in $\mathrm{DM}$ patients.

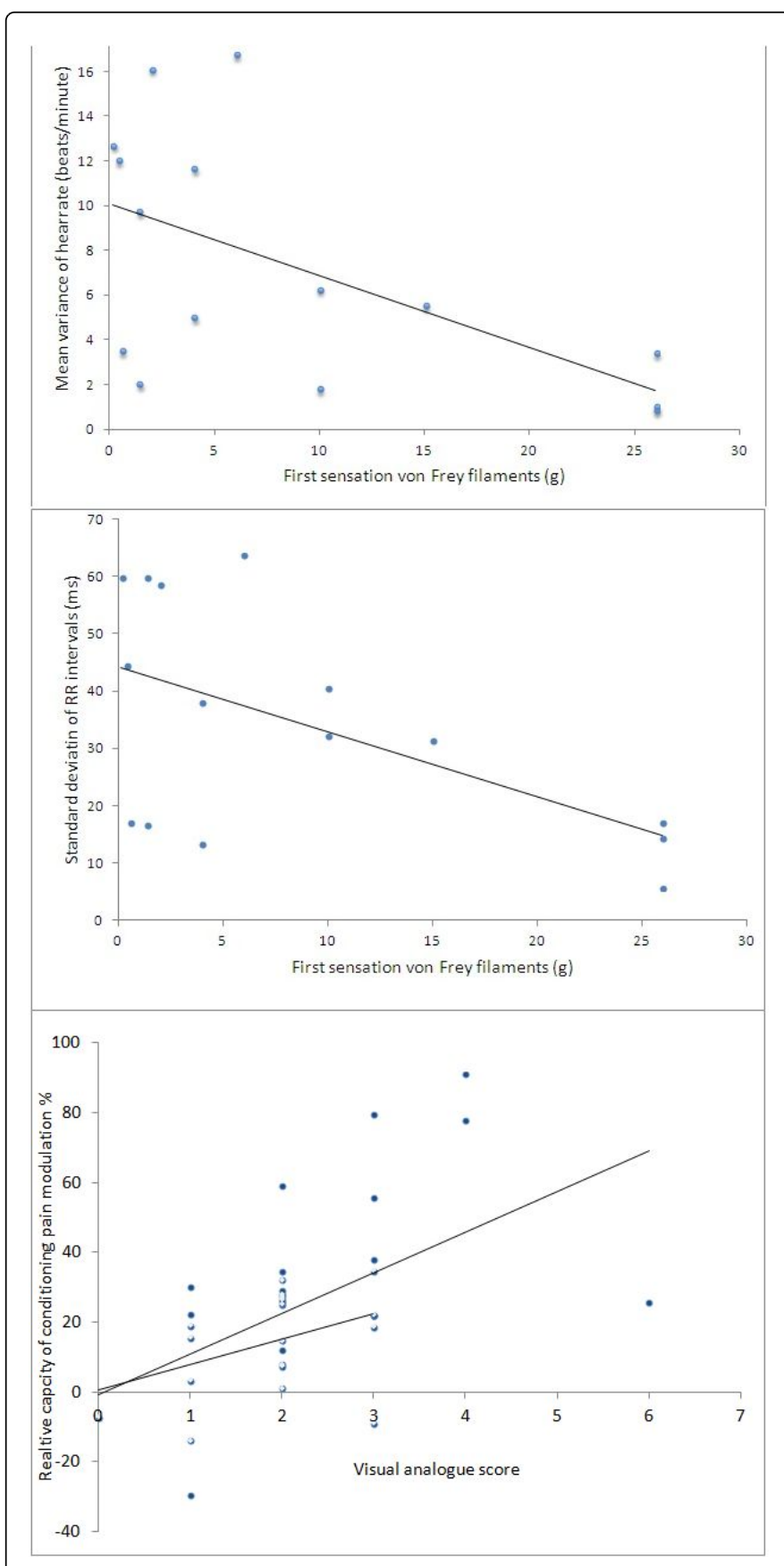

Figure 3: Tactile detection of Von Frey monofilament was negatively associated to autonomic neuropathy shown as mean variance of heart rate (beats $/ \mathrm{min}$ ) in the top panel, and to the standard deviation of the mean RR interval (ms) in the middle panel. The lower panel shows association between efficacy of descending pain modulation and the sensory response to pinprick, done with a 26 gr. von Frey monofilament. 


\section{Clinical correlations}

Associations between von Frey tactile detection threshold and cardiac autonomic parameters in patients are shown in Figure 3. Significant negative correlations were found to both mean variance in heart rate $(\mathrm{r}=-0.63, \mathrm{P}=0.01)$ and mean standard deviation of heart rate $(\mathrm{r}=-0.69, \mathrm{P}=0.004)$.

In the combined cohort of patients and healthy volunteers, an association between sensory score to pinprick and efficacy of CPM was found $(\mathrm{r}=0.52, \mathrm{P}=0.001)$, which also was present in the patient population $(\mathrm{r}=0.36, \mathrm{P}=0.048)$.

\section{Discussion}

Patients with longstanding DM showed evidence of generalized neuronal damage manifested as sensorimotor, autonomic and central neuropathies. For the first time reported, to our best knowledge, it was shown that the degree of peripheral hypoesthesia was associated with heart rate variability and impaired conditioned pain modulation.

\section{Diabetes induced neuropathies}

Diabetes induced neuronal damage leads directly and indirectly to neurodegeneration, which present as Schwann cell dysfunction, decreased neurotransmission and diminished neurotrophic axonal support [21]. However, the generalized co-existing glia cell activation and neuroinflammation is often under-diagnosed.

CPM influences primarily dorsal horn convergent neurons through a delicate balance involving descending pathways from brainstem nuclei. In comparison to $\mathrm{HV}, \mathrm{DM}$ patients in the present study showed non-dynamic and less efficacious CPM, which could indicate dysfunctional brainstem regulation. These findings could be supported by the fact that a similar group of DM patients have previously shown altered brain processing -likely centrally induced neuropathy- evident as a delay in the activation of a specific brain network involving brainstem/operculum/frontal cortex and finally, microstructural neurodegenerative changes in pain specific brain areas of DM patients have been identified by use of diffusion tension imaging [23].

Impairment of CPM are previously reported in chronic painful conditions such as fibromyalgia, painful osteoarthritis, temporomandibular joint disease, chronic low back pain, inflammatory bowel disease and chronic pancreatitis, where it is considered to predispose development and persistence of chronic pain [24-30]. DM patients with clinical suspicion of sensorimotor neuropathy may have concomitant peripheral $\mathrm{A} \delta$ - and C-neuropathy and consequently decreased afferent transmission. Therefore, it must be considered if the observed changes in CPM are caused by deafferentation. If so, the decreased involvement of convergent dorsal horn neurons would lead to lead to CPM patterns which could be misinterpreted as central impairment. However, in this study there was no difference in sensory response between patients and HV to von Frey pinprick and muscle pressure at baseline (indicating comparable afferent input), it is plausible that concerns regarding alterations inafferent transmission may be ruled out.

Taken together, DM patients with clinical suspicion of peripheral neuropathy in this study showed co-existence of autonomic and altered central processing, which gives rise to speculate whether diabetes patients more often than expected, suffer from generalized polyneuropathy. In that case, future treatment possibilities could include agents which directly increase anti-oxidative capacity, support neurotrophic properties or enhance reparative processes after injury $[21,31]$.

\section{Methodological considerations}

Patient recruitment was based on clinical suspicion of diabetes evident as peripheral sensorimotor neuropathy, however not all patients fulfilled the Toronto criteria, which was put forward after the study onset, and hence only a portion of the patients had had nerve conduction velocities assessed. HRV was assessed and interpreted without controlling for hypertension [2]. As the patient group has higher prevalence of hypertension than healthy, the study can be criticized for having interpreted HRV in the groups without controlling for possible confounding by hypertension. With only 19 subjects, data showed larger variety than expected, which gave rise to speculate whether the study was underpowered. Indeed the findings must be interpreted cautiously. However, even with this small study group, consistent differences between patients and HV were found, and thus we believe that the obtained results are fairly applicable in diabetes patients with sensorimotor neuropathy.

Quantitative Sensory Testing (QST) is psychophysical in nature with an objective physical stimulus (von Frey monofilaments or pressure algometry) and a subjective report as the response [8]. Hence, QST may be too dependent on compliant cooperation from the subject, otherwise possibly biases the outcome. Furthermore, even though the Von Frey filament endings are rounded to avoid $A \beta$ nociceptor activation, first tactile detection activates non-specific nociceptors as well as low threshold mechanoreceptors, possibly blurring the sensation [32]. Nevertheless, hypoesthesia was convincingly shown at the dorsum of the first toe on the dominant foot. In DM patients CPM induction has, to the best of our knowledge, not been induced by the cold pressor test, even though it has been widely used to explore central pain mechanisms in other diseases and in healthy controls $[29,30]$. The handheld pressure algometer has previously been validated, and to familiarize the subjects with the scoring procedure, participants were trained by applying muscle pressure in the femoral quadriceps muscle [33,34]. All patients and HV withstood immersion of their hand in 180 seconds. This likely reflect that these DM patients did not experience neuropathic pain as it is in contrast to chronic pain patients who cannot withstand the complete immersion in the cold pressor test, likely because they have developed central sensitization [29]. On the other hand, the finding raised speculations whether DM patients with small fiber neuropathy perceived less conditioning pain, resulting in less efficacious CPM. However, no differences between patients and HV were found in perceived pain during the cold pressor, and hence we believe CPM was sufficiently induced.

\section{Conclusion}

DM patients with suspicion of sensorimotor neuropathy showed generalized neuronal damage evident as peripheral hypoesthesia, autonomic neuropathy and impaired conditioned pain modulation. Increased Von Frey tactile detection threshold were associated with reduced heart rate variability and reduced efficacy of CPM. The clinically applicable platform presented in the present study may be used as a prognostic tool to quantitatively evaluate the extension, severity and progression of diabetes induced neuropathy. 


\section{Author Contribution}

Study design: CB, AMD, LAN; Data collection: ES, CB, JBF; Data analysis: $\mathrm{CB}$, ES; Interpretation of results: All co-authors; Manuscript preparation: $\mathrm{CB}, \mathrm{LAN}$, Provided funding: $\mathrm{CB}, \mathrm{AMD}$; Critical revision of manuscript: All co-authors

\section{Acknowledgment}

Dr. Christina Brock is the guarantor of this work and, as such, had full access to all the data in the study and takes responsibility for the integrity of the data and the accuracy of the data analysis.

The research leading to these results has received funding from the European Community's Seventh Framework Programme FP7/2007-2013 under grant agreement no. 223630. No other conflicts of interest relevant to this article were reported.

\section{References}

1. American Diabetes Association (2014). Diagnosis and Classification of Diabetes Mellitus. Daibetes Care 37: Supplement 1,S81-S90.

2. Tesfaye S, Boulton AJ, Dyck PJ, Freeman R, Horowitz M, et al. (2010) Diabetic neuropathies: update on definitions, diagnostic criteria, estimation of severity, and treatments. Diabetes Care 33: 2285-2293.

3. Martyn CN, Hughes RA (1997) Epidemiology of peripheral neuropathy. J Neurol Neurosurg Psychiatry 62: 310-318.

4. Hovaguimian A, Gibbons CH (2011) Clinical Approach to the Treatment of Painful Diabetic Neuropathy. Ther Adv Endocrinol Metab 2: 27-38.

5. Hovaguimian A, Gibbons CH (2011) Diagnosis and treatment of pain in small-fiber neuropathy. Curr Pain Headache Rep 15: 193-200.

6. Ziegler D, Rathmann W, Dickhaus T, Meisinger C, Mielck A; KORA Study Group (2009) Neuropathic pain in diabetes, prediabetes and normal glucose tolerance: the MONICA/KORA Augsburg Surveys S2 and S3. Pain Med 10: 393-400.

7. Askwith T, Zeng W, Eggo MC, Stevens MJ (2009) Oxidative stress and dysregulation of the taurine transporter in high-glucose-exposed human Schwann cells: implications for pathogenesis of diabetic neuropathy. Am J Physiol Endocrinol Metab 297: E620-628.

8. Rolke R, Baron R, Maier C, Tölle TR, Treede RD, et al. (2006) Quantitative sensory testing in the German Research Network on Neuropathic Pain (DFNS): standardized protocol and reference values. Pain 123: 231-243.

9. England JD, Gronseth GS, Franklin G, Carter GT, Kinsella LJ, et al. (2009) Practice Parameter: evaluation of distal symmetric polyneuropathy: role of autonomic testing, nerve biopsy, and skin biopsy (an evidence-based review). Report of the American Academy of Neurology, American Association of Neuromuscular and Electrodiagnostic Medicine, and American Academy of Physical Medicine and Rehabilitation. Neurology 72: 177-184.

10. Shtein RM, Callaghan BC (2013) Corneal confocal microscopy as a measure of diabetic neuropathy. Diabetes 62: 25-26.

11. Tavakoli M, Petropoulos IN, Malik RA (2013) Corneal confocal microscopy to assess diabetic neuropathy: an eye on the foot. J Diabetes Sci Technol 7: 1179-1189.

12. Low PA, Benrud-Larson LM, Sletten DM, Opfer-Gehrking TL, Weigand SD, et al. (2004) Autonomic symptoms and diabetic neuropathy: a population-based study. Diabetes Care 27: 2942-2947.

13. Brock C, Olesen SS, Valeriani M, Arendt-Nielsen L, Drewes AM (2012) Brain activity in rectosigmoid pain: unravelling conditioning pain modulatory pathways. Clin Neurophysiol 123: 829-837.

14. Brock C, Søfteland E, Gunterberg V, Frøkjær JB, Lelic D, et al. (2013) Diabetic autonomic neuropathy affects symptom generation and braingut axis. Diabetes Care 36: 3698-3705.
15. Lelic D, Brock C, Simren M, Frokjaer JB, Softeland E, et al. (2014) The brain networks encoding visceral sensation in patients with gastrointestinal symptoms due to diabetic neuropathy. Neurogastroenterol Motil 26: 46-58.

16. Yarnitsky D, Granot M, Nahman-Averbuch H, Khamaisi M, Granovsky Y (2012) Conditioned pain modulation predicts duloxetine efficacy in painful diabetic neuropathy. Pain 153: 1193-1198.

17. Søfteland E, Brock C, Frøkjær JB, Brøgger J, Madácsy L, et al. (2014) Association between visceral, cardiac and sensorimotor polyneuropathies in diabetes mellitus. J Diabetes Complications 28: 370-377.

18. Boulton AJ, Vinik AI, Arezzo JC, Bril V, Feldman EL, et al. (2005) Diabetic neuropathies: a statement by the American Diabetes Association. Diabetes Care 28: 956-962.

19. Drewes AM, Schipper KP, Dimcevski G, Petersen P, Andersen OK, et al. (2002) Multimodal assessment of pain in the esophagus: a new experimental model. Am J Physiol Gastrointest Liver Physiol 283: G95-103.

20. Novak V, Saul JP, Eckberg DL (1997) Task Force report on heart rate variability. Circulation 96: 1056-1057.

21. Eckersley L (2002) Role of the Schwann cell in diabetic neuropathy. Int Rev Neurobiol 50: 293-321.

22. Lelic D, Brock C, Søfteland E, Frøkjær JB, Andresen T, et al. (2013) Brain networks encoding rectal sensation in type 1 diabetes. Neuroscience 237: 96-105.

23. Frøkjær JB, Andersen LW, Brock C, Simrén M, Ljungberg M, et al. (2013) Altered brain microstructure assessed by diffusion tensor imaging in patients with diabetes and gastrointestinal symptoms. Diabetes Care 36: 662-668.

24. Lautenbacher S, Rollman GB (1997) Possible deficiencies of pain modulation in fibromyalgia. Clin J Pain 13: 189-196.

25. Kosek E, Ordeberg G (2000) Lack of pressure pain modulation by heterotopic noxious conditioning stimulation in patients with painful osteoarthritis before, but not following, surgical pain relief. Pain 88: 69-78.

26. Bragdon EE, Light KC, Costello NL, Sigurdsson A, Bunting S, et al. (2002) Group differences in pain modulation: pain-free women compared to pain-free men and to women with TMD. Pain 96: 227-237.

27. Peters ML, Schmidt AJ, Van den Hout MA, Koopmans R, Sluijter ME (1992) Chronic back pain, acute postoperative pain and the activation of diffuse noxious inhibitory controls (DNIC). Pain 50: 177-187.

28. Wilder-Smith CH, Robert-Yap J (2007) Abnormal endogenous pain modulation and somatic and visceral hypersensitivity in female patients with irritable bowel syndrome. World J Gastroenterol 13: 3699-3704.

29. Olesen SS, Brock C, Krarup AL, Funch-Jensen P, Arendt-Nielsen L, et al. (2010) Descending inhibitory pain modulation is impaired in patients with chronic pancreatitis. Clin Gastroenterol Hepatol 8: 724-730.

30. Pud D, Granovsky Y, Yarnitsky D (2009) The methodology of experimentally induced diffuse noxious inhibitory control (DNIC)-like effect in humans. Pain 144: 16-19.

31. Mahmood D, Singh BK, Akhtar M (2009) Diabetic neuropathy: therapies on the horizon. J Pharm Pharmacol 61: 1137-1145.

32. Le Bars D, Gozariu M, Cadden SW (2001) Animal models of nociception. Pharmacol Rev 53: 597-652.

33. Finocchietti S, Andresen T, Arendt-Nielsen L, Graven-Nielsen T (2012) Pain evoked by pressure stimulation on the tibia bone - influence of probe diameter on tissue stress and strain. Eur J Pain 16: 534-542.

34. Andresen T, Pfeiffer-Jensen M, Brock C, Drewes AM, Arendt-Nielsen L (2013) A human experimental bone pain model. Basic Clin Pharmacol Toxicol 112: 116-123. 